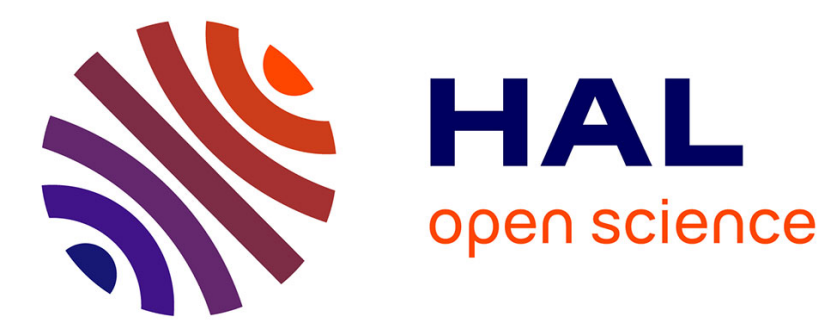

\title{
Stability Analysis of a Longitudinal Control Law for Autonomous Vehicles
}

\author{
Alain Girault, Sergio Yovine
}

\section{To cite this version:}

Alain Girault, Sergio Yovine. Stability Analysis of a Longitudinal Control Law for Autonomous Vehicles. RR-3498, INRIA. 1998. inria-00073187

\section{HAL Id: inria-00073187 https://hal.inria.fr/inria-00073187}

Submitted on 24 May 2006

HAL is a multi-disciplinary open access archive for the deposit and dissemination of scientific research documents, whether they are published or not. The documents may come from teaching and research institutions in France or abroad, or from public or private research centers.
L'archive ouverte pluridisciplinaire HAL, est destinée au dépôt et à la diffusion de documents scientifiques de niveau recherche, publiés ou non, émanant des établissements d'enseignement et de recherche français ou étrangers, des laboratoires publics ou privés. 
Stability Analysis of a Longitudinal Control Law for Autonomous Vehicles

Alain Girault, Sergio Yovine

$$
\mathbf{N}^{\circ} 3498
$$

Septembre 1998

THÈME 4 



\title{
Stability Analysis of a Longitudinal Control Law for Autonomous Vehicles
}

\author{
Alain Girault* , Sergio Yovine ${ }^{\dagger}$ \\ Thème 4 - Simulation et optimisation \\ de systèmes complexes \\ Projet BIP \\ Rapport de recherche $\mathrm{n}^{\circ} 3498$ - Septembre 1998 - 20 pages
}

\begin{abstract}
We focus in this article on the analysis of an existing acceleration law for a following autonomous vehicle with constant time headway goal. We first define a function that measures the positioning of the following vehicle compared to the leading vehicle with respect to the desired time headway. We study the convergence and bounds of this positioning function and prove some key results in an ideal case, i.e., when all accelerations are unbounded. Then we conduct several simulations that validate our theoretical results and show their limitations in real cases, i.e., when the accelerations are technologically bounded. Finally we use decoupling and feedback linearization techniques to derive a new acceleration law whose convergence is always exponential in the ideal case, and we compare both laws.
\end{abstract}

Key-words: Acceleration law, asymptotic stability, autonomous intelligent cruise control, autonomous vehicles, automated highway, constant time headway, longitudinal control, micro-simulation, saturation constrains.

(Résumé : tsvp)

* InRIA-Bip, Alain.Girault@inrialpes.fr.

$\dagger$ California-PATH and VÉRIMAG, Sergio.Yovine@imag.fr, Partially supported by NSF grant ECS-9725148.

Unité de recherche INRIA Rhône-Alpes

655, avenue de l'Europe, 38330 MONTBONNOT ST MARTIN (France)

Téléphone : 0476615200 - International: +33476615200

Télécopie : 0476615252 - International: +33476615252 


\section{Analyse de stabilité d'une loi de contrôle longitudinal pour véhicules autonomes}

Résumé : Nous nous intéressons dans cet article à l'analyse d'une loi d'accélération existante pour véhicules autonomes. La stratégie de contrôle consiste à maintenir un délai frontal constant avec le véhicule leader. Nous définissons tout d'abord une fonction qui évalue le positionnement du véhicule suiveur par rapport au véhicule leader et au délai frontal désiré. Nous étudions la convergence et les bornes de cette fonction de positionnement et établissons plusieurs résultats clé dans le cas idéal, c'est-à-dire quand les accélérations des véhicules ne sont pas bornées. Puis nous procédons à des simulations qui valident nos résultats théoriques et qui montrent leurs limitations dans le cas réel, c'est-à-dire quand les accélérations sont bornées par les limites technologiques. Enfin nous appliquons des techniques de découplage et de linéarisation afin de calculer une nouvelle loi d'accélération dont la convergence est exponentielle dans le cas idéal. Nous comparons les deux lois d'accélération et nous concluons.

Mots-clé : Loi d'accélération, stabilité asymptotique, véhicules autonomes, route automatisée, délai frontal constant, contrôle longitudinal, micro-simulation, contraintes de saturation. 


\section{Introduction}

Passenger safety and traffic congestion are growing problems in several urban corridors. Two approaches can be used to alleviate these problems: construction of more lanes on congested highways or use of emerging technologies for more automated traffic control. The first approach, constructing more lanes or highways, is rapidly becoming untenable because of lack of right of ways, complexity of highway design and layout, costs and environmental considerations, and so on. Hence several metropolitan transportation agencies are turning towards higher levels of automation technologies to address these problems.

Different automation strategies have been proposed in $[1,8]$. They are classified according to their distribution of intelligence and cooperative attributes. In this paper, we address the case where all automation intelligence is concentrated within individual vehicles, leading to the so called autonomous scenario, where there is no communication between vehicles and the infrastructure. Finally, vehicles are equipped with several sensors, allowing them to perceive the speed and position of the nearby vehicles. This system is known as AICC, for "Autonomous Intelligent Cruise Control."

Our starting point is a traffic simulation that was conducted for the Houston Metro $[2,3]$. We simulated $10 \mathrm{kms}$ of a single lane freeway, approximately modeled after the Katy Corridor of Interstate Highway 10. We considered two traffic densities, a low density of 1800 vehicles/hour, and a high density of 4000 vehicles/hour. Vehicles were autonomous, i.e., there was no communication with other vehicles or with the infrastructure. Each vehicle had an autonomous controller in charge of insuring the correct insertion of the vehicle at the merge junction, as well as preventing collisions with other nearby vehicles. For this purpose, we designed an acceleration control law, and we empirically tuned its parameters. This allowed us to achieve a no-collision simulation, even in the high density case. In this paper, we study analytically this control law and we prove some key stability results. Specifically, we prove that under some dynamic condition, the vehicles never collide. We first conduct this analysis in the non-saturated case, and then in the saturated case.

\section{Vehicle controller}

The vehicles are autonomous, i.e., they do not communicate with each other and sensor range is limited. Thus, at any time a vehicle knows which vehicles are in front of and behind him, both in its own lane and in the side lane. The longitudinal speed of each vehicle within sensor range is also known. Each vehicle has its own controller that outputs the current longitudinal acceleration and the lateral speed. Then the kinematic model is a basic 2 dimensions model.

The controller will naturally have several modes, and within each mode will have a continuous behavior. On the other hand, switching to one mode to another one is a discrete evolution. Modes are linked to the environmental situation of the given vehicle: is there a vehicle in front, is the vehicle trying to merge in the traffic, is the vehicle yielding to another vehicle, and so on. This combination of a continuous behavior and a discrete behavior makes the controller a hybrid system.

In this paper we focus on the continuous behavior of the controller. Precisely, we wish to study the stability of the control law. For this purpose, we restrict our case study to two vehicles driving along a single lane straight highway. The behavior of the leading vehicle is fixed and consists in alternatively

$\mathrm{RR} \mathrm{n}^{\circ} 3498$ 
braking and accelerating (within technology bounds). The following vehicle drives right behind the leading vehicle while trying to avoid collisions, and its behavior is dictated by an acceleration control law. There exist three basic classes of such control laws that vary according to the desired following distance between the leading and the following vehicle [6]:

- Constant spacing: such systems are most suitable for use at very short spacings, particularly within platoons of vehicles. However, they require information about the acceleration of the leading vehicle as well as inter-vehicle communication.

- Constant time headway: such systems use only information derived from sensors, and they maintain spacing proportional to the speed $v$.

- Constant safety factor: such systems are designed so that the instantaneous stopping of the leading vehicle does not cause the following vehicle to collide with it. Typically, they maintain spacing proportional to $v^{2}$.

For these reasons, we choose an acceleration law that maintains a constant time headway. We present this acceleration law in the next Section.

\section{Design of the acceleration law}

At any time $t$, the leading vehicle and the following vehicle are defined by the following data:

\begin{tabular}{|l|c|c|}
\hline data & leading vehicle & following vehicle \\
\hline acceleration & $a_{l}(t)$ & $a_{f}(t)$ \\
velocity & $v_{l}(t)$ & $v_{f}(t)$ \\
position & $p_{l}(t)$ & $p_{f}(t)$ \\
\hline
\end{tabular}

At any time the acceleration must remain within technology bounds. The maximum braking $a_{m i n}$ is set to $-0.5 g$, while the maximum acceleration $a_{\max }$ is set to $+0.2 g$, and $g=9.81 \mathrm{~ms}^{-2}$.

The aim of the controller is to try to keep a constant time headway $h$. We thus compute the time before impact, i.e., the gap divided by the vehicle speed. We then compute the ratio of the time before impact by the time headway. This ratio $r(t)$ is a pure number in the range $[0,+\infty)$ :

$$
r(t)=\frac{p_{l}(t)-p_{f}(t)}{h v_{f}(t)}
$$

The ratio measures the correct positioning of the following vehicle relatively to the leading vehicle: a value between 0 and 1 means that the following vehicle is too close to the leading vehicle and must brake. A value greater than 1 means that the following vehicle is too far from the leading vehicle and can accelerate; a value equal to 1 means that the following vehicle is exactly at the desired time headway from the leading vehicle. We therefore choose an acceleration proportional to the positioning error:

$$
a_{f}(t)=\lambda(r(t)-1)
$$


To achieve a faster adaptation to the driving of the leading vehicle, we also take into account the relative speed:

$$
a_{f}(t)=\lambda(r(t)-1)+\mu\left(v_{l}(t)-v_{f}(t)\right)
$$

Here $\lambda$ and $\mu$ are two positive parameters, respectively of dimension $m s^{-2}$ and $s^{-1}$. Following [4], we can compute the Laplace form which leads to take $\mu=1 / h$. Therefore:

$$
a_{f}(t)=\lambda(r(t)-1)+\frac{v_{l}(t)-v_{f}(t)}{h}
$$

The value of the desired time headway $h$ has a direct influence on the maximal density that can be accommodated by an automated highway. Intuitively, for a given density and nominal speed, it is possible to compute the average gap between two successive vehicles. In [2], we have conducted extensive simulations of an automated highway which show that this gap divided by the nominal speed is the time headway that will not cause congestion. The current technology on sensors, actuators, and computing systems allows a time headway as low as $0.3 \mathrm{~s}$.

Our acceleration law is intuitively satisfying: when the following vehicle is too close it brakes, while when it is too far it accelerates. We now wish to prove that we avoid accidents, that is, we keep the ratio strictly greater than 0 . Since $v_{f}(t)=p_{f}^{\prime}(t)$ and $a_{f}(t)=p_{f}^{\prime \prime}(t)$, we can rewrite equation (4) as:

$$
p_{f}^{\prime \prime}(t)=\frac{\lambda\left(p_{l}(t)-p_{f}(t)-h p_{f}^{\prime}(t)\right)+p_{f}^{\prime}(t)\left(v_{l}(t)-p_{f}^{\prime}(t)\right)}{h p_{f}^{\prime}(t)}
$$

which yields:

$$
h p_{f}^{\prime}(t) p_{f}^{\prime \prime}(t)+\lambda p_{f}(t)+\lambda h p_{f}^{\prime}(t)-v_{l}(t) p_{f}^{\prime}(t)+p_{f}^{\prime}(t)^{2}=\lambda p_{l}(t)
$$

The differential equation (6) is not integrable. So it is not possible to study analytically the variations of $p_{f}(t)$. Besides, according to equation (1), the variations of the ratio depend on the variations of $a_{l}(t)$, on which we can make no assumption. Our idea is therefore to study analytically the $t \mapsto r(t)$ function rather that the $t \mapsto p_{f}(t)$.

In the first part of this article, we establish some key results on the asymptotic stability of our acceleration law. In particular, we give a dynamic condition on the gain $\lambda$ under which the ratio $r(t)$ converges towards 1 . We then prove that when $r(t)=1$, if $a_{l}(t)$ is bounded then $a_{f}(t)$ is also bounded by the same bounds. In the second part, we present the results of several simulations which indicate the limit of what can be proved in the general case. We always assume that $a_{l}(t) \in[-0.5 g,+0.2 g]$. We first study the case where $a_{f}$ is unsaturated, i.e., $a_{f}(t) \in(-\infty,+\infty)$, and then we also saturate $a_{f}$ to the technology bounds, i.e., $a_{f}(t) \in[-0.5 g,+0.2 g]$. In the last part, we apply decoupling and feedback linearization techniques to derive a new acceleration law whose convergence is always exponential in the ideal case, i.e., $a_{f}(t) \in(-\infty,+\infty)$. We compare both accelerations laws and draw some conclusions. Throughout this article, we assume that the jerks on $a_{l}(t)$ and $a_{f}(t)$ are potentially infinite.

Similar work on asymptotic stability analysis of dynamic systems with saturation constrains include $[9,7]$. However these works deal with linear and second order dynamic systems, so their results are not transposable to our problem.

$\mathrm{RR} \mathrm{n}^{\circ} 3498$ 


\section{The ratio function $t \mapsto r(t)$}

We first study the variations of the ratio function. This leads us to distinguish three exclusive cases: $r(t) \in[0,1), r(t)=1$, and $r(t) \in(1,+\infty)$. We then prove that in both cases where $r(t) \neq 1$, there exists a limit when $t \rightarrow+\infty$. We prove that in both cases the limit is equal to 1 . Finally, we prove that in the $r(t)=1$ case, if the acceleration of the leading vehicle is bounded, i.e., $a_{l}(t) \in\left[a_{\min }, a_{\max }\right]$, then the acceleration of the following vehicle is also bounded, i.e., $a_{f}(t) \in\left[a_{\min }, a_{\max }\right]$.

\subsection{Study of the variations of $r(t)$}

We restrict ourselves to the case where $v_{f}(t) \neq 0$, i.e., the following vehicle is moving. Since we consider automatic highway applications, we can also assume that both vehicles are moving forward. Thus we assume $v_{f}(t)>0$ and $v_{l}(t)>0$. Under this assumption, the $t \mapsto r(t)$ function is continuous and infinitely derivable, and its derivatives are also continuous: we say that $r$ is a $\mathcal{C}^{\infty}$-function. Its first derivative is:

$$
\begin{aligned}
r^{\prime}(t) & =\frac{h v_{f}(t)\left(v_{l}(t)-v_{f}(t)\right)-h a_{f}(t)\left(p_{l}(t)-p_{f}(t)\right)}{h^{2} v_{f}(t)^{2}} \\
& =\frac{v_{l}(t)-v_{f}(t)}{h v_{f}(t)}-\frac{a_{f}(t) r(t)}{v_{f}(t)} \\
& =\frac{v_{l}(t)-v_{f}(t)}{h v_{f}(t)}-\frac{\lambda r(t)(r(t)-1)}{v_{f}(t)}-\frac{r(t)\left(v_{l}(t)-v_{f}(t)\right)}{h v_{f}(t)} \\
& =\frac{v_{l}(t)-v_{f}(t)}{h v_{f}(t)}(1-r(t))-\frac{\lambda r(t)}{v_{f}(t)}(r(t)-1) \\
& =\frac{1-r(t)}{v_{f}(t)}\left(\frac{v_{l}(t)-v_{f}(t)}{h}+\lambda r(t)\right) \\
& =\frac{(1-r(t)) X_{0}^{0}(t)}{v_{f}(t)}
\end{aligned}
$$

where

$$
X_{0}^{0}(t)=\frac{v_{l}(t)-v_{f}(t)}{h}+\lambda r(t)=a_{f}(t)+\lambda
$$

Replacing $X_{0}^{0}(t)$ in equation (7) yields:

$$
r^{\prime}(t)=\frac{\left(1-r(t)\left(a_{f}(t)+\lambda\right)\right.}{v_{f}(t)}
$$

For any $t_{0}$ such that $r\left(t_{0}\right)=1$, we have $r^{\prime}\left(t_{0}\right)=0$. Our goal is to prove that $\forall n, r^{(n)}\left(t_{0}\right)=0$. First we compute the second derivative of $r$ : 


$$
\begin{aligned}
r^{\prime \prime}(t) & =\frac{-r^{\prime}(t) X_{0}^{0}(t) v_{f}(t)+(1-r(t)) X_{0}^{0 \prime}(t) v_{f}(t)-(1-r(t)) X_{0}^{0}(t) a_{f}(t)}{v_{f}(t)^{2}} \\
& =\frac{(1-r(t))\left(X_{0}^{0^{\prime}}(t) v_{f}(t)-X_{0}^{0}(t) a_{f}(t)\right)-r^{\prime}(t) X_{0}^{0}(t) v_{f}(t)}{v_{f}(t)^{2}} \\
& =\frac{(1-r(t)) X_{1}^{0}(t)+r^{\prime}(t) X_{1}^{1}(t)}{v_{f}(t)^{2}}
\end{aligned}
$$

where

$$
\left\{\begin{array}{l}
X_{1}^{0}(t)=X_{0}^{0}(t) v_{f}(t)-X_{0}^{0}(t) a_{f}(t) \\
X_{1}^{1}(t)=-X_{0}^{0}(t) v_{f}(t)
\end{array}\right.
$$

We now prove by induction that the $n^{\text {th }}$ derivative is:

$$
r^{(n)}(t)=\frac{(1-r(t)) X_{n-1}^{0}(t)+\sum_{i=1}^{n-1} r^{(i)}(t) X_{n-1}^{i}(t)}{v_{f}(t)^{2^{n-1}}}
$$

This is true for $n=0$ and $n=1$. The computation of the $n+1^{\text {th }}$ derivative is left in annex. Therefore, if $\exists t_{0}$ such that $r\left(t_{0}\right)=1$, then we have:

- $r\left(t_{0}\right)=1 \Rightarrow r^{\prime}\left(t_{0}\right)=0$

- $r\left(t_{0}\right)=1 \wedge r^{\prime}\left(t_{0}\right)=0 \Rightarrow r^{\prime \prime}\left(t_{0}\right)=0$,

- $r\left(t_{0}\right)=1 \wedge r^{\prime}\left(t_{0}\right)=0 \wedge r^{\prime \prime}\left(t_{0}\right)=0 \Rightarrow r^{\prime \prime \prime}\left(t_{0}\right)=0$,

- and so on.

So if $\exists t_{0}$ such that $r\left(t_{0}\right)=1$, then $\forall n, r^{(n)}\left(t_{0}\right)=0$. Moreover, a $\mathcal{C}^{\infty}$-function such that all its derivatives are null in one point is a constant function. Therefore, the following proposition holds:

Proposition 1 If $\exists t_{0}$ such that $r\left(t_{0}\right)=1$, then $\forall t, r(t)=1$.

Corollary 1 The ratio function $t \mapsto r(t)$ is such that:

- $\forall t, r(t) \in[0,1)$, or

- $\forall t, r(t)=1$, or

- $\forall t, r(t) \in(1,+\infty)$.

Now let us study the variations of the $t \mapsto r(t)$ function. According to equation (9), if $a_{f}(t)>-\lambda$, then we have the two following variation tables:

$\mathrm{RR} \mathrm{n}^{\circ} 3498$ 

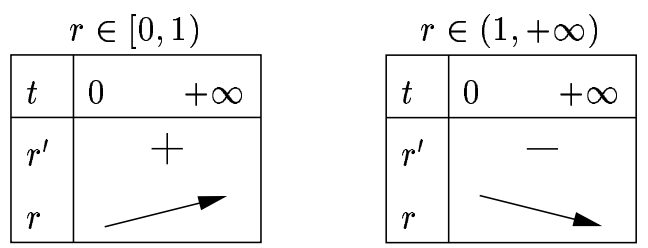

This is an important result since it establishes that, under the condition $a_{f}(t)>-\lambda$, the ratio evolves in the good direction: if the following vehicle is too close, i.e., $r(t)<1$, then the ratio increases, while if the following vehicle is far away, i.e., $r(t)>1$, then the ratio decreases.

\subsection{Study of the limit of $r(t)$}

Throughout this Section, we suppose that $a_{f}(t)>-\lambda$.

In the $r \in[0,1)$ case, since the function $t \mapsto r(t)$ is increasing and bounded, it has a limit, say l. Similarly, in the $r \in(1,+\infty)$ case, since the function $t \mapsto r(t)$ is decreasing and bounded, it has a limit, say $L$. In summary:

- If $r \in[0,1)$ then $\lim _{t \rightarrow+\infty} r(t)=l \leq 1$.

- If $r \in(1,+\infty)$ then $\lim _{t \rightarrow+\infty} r(t)=L \geq 1$.

Now let us prove that $l=1$. Since $\lim _{t \rightarrow+\infty} r(t)$ is finite, then it is an asymptotic limit and therefore $\lim _{t \rightarrow+\infty} r^{\prime}(t)=0$. According to equation (9), either $\lim _{t \rightarrow+\infty}(1-r(t))=1-l=0$, or $\lim _{t \rightarrow+\infty}\left(a_{f}(t)+\lambda\right)=0$, or $\lim _{t \rightarrow+\infty} v_{f}(t)= \pm \infty$. Let us suppose that $l<1$.

If $\lim _{t \rightarrow+\infty}\left(a_{f}(t)+\lambda\right)=0$, then $\lim _{t \rightarrow+\infty} v_{f}(t)=-\infty$. This must hold whatever be the behavior of $a_{l}(t)$ and $v_{l}(t)$. Since $\lim _{t \rightarrow+\infty} r(t)=l$, it is impossible. The case where $\lim _{t \rightarrow+\infty} v_{f}(t)= \pm \infty$ is analogous. As a consequence, our hypothesis $l<1$ is false. Hence $l=1$.

The proof that $L=1$ is similar. We thus have the following result:

Proposition 2 If $\exists t_{0}: \forall t>t_{0}, a_{f}(t)>-\lambda$, then $\lim _{t \rightarrow+\infty} r(t)=1$.

This is a major result since it establishes that, under the condition $a_{f}(t)>-\lambda$, the following vehicle never collides with the leading vehicle!

\subsection{Study of the bounds of $r(t)$}

We assume that the acceleration of the leading vehicle is bounded, i.e., $a_{l}(t) \in\left[a_{\min }, a_{\max }\right]$. According to our technology bounds, $a_{\min }=-0.5 g$ and $a_{\max }=+0.2 g$. We also assume that $r(t)=1$. Then the acceleration of the following vehicle and its derivative are: 


$$
\begin{aligned}
& a_{f}(t)=\frac{v_{l}(t)-v_{f}(t)}{h} \\
& a_{f}^{\prime}(t)=\frac{a_{l}(t)-a_{f}(t)}{h}
\end{aligned}
$$

Equation (13) is a differential equation of the form:

$$
a_{f}^{\prime}(t)+\frac{a_{f}(t)}{h}=\frac{a_{l}(t)}{h}
$$

Its general solution is:

$$
a_{f}(t)=\frac{e^{-t / h}}{h}\left(\int_{0}^{t} e^{x / h} a_{l}(x) d x+k h\right)
$$

The initial conditions give us:

$$
a_{f}(0)=\frac{1}{h}(0+k h)=k
$$

Now since $a_{l}(t) \in\left[a_{\min }, a_{\max }\right]$, we have:

$$
\begin{aligned}
& a_{\min } \leq \quad a_{l}(x) \quad \leq a_{\max } \\
& \Leftrightarrow e^{x / h} a_{\min } \leq \quad e^{x / h} a_{l}(t) \quad \leq e^{x / h} a_{\max } \\
& \Leftrightarrow \int_{0}^{t} e^{x / h} a_{\min } d x \leq \quad \int_{0}^{t} e^{x / h} a_{l}(x) d x \quad \leq \int_{0}^{t} e^{x / h} a_{\max } d x \\
& \Leftrightarrow\left[h e^{x / h} a_{\min }\right]_{0}^{t} \leq \quad \int_{0}^{t} e^{x / h} a_{l}(x) d x \quad \leq\left[h e^{x / h} a_{\max }\right]_{0}^{t} \\
& \Leftrightarrow h e^{t / h} a_{\min }-h a_{\min } \leq \quad \int_{0}^{t} e^{x / h} a_{l}(x) d x \quad \leq h e^{t / h} a_{\max }-h a_{\max } \\
& \Leftrightarrow h e^{t / h} a_{\min }-h a_{\min }+h a_{f}(0) \leq \int_{0}^{t} e^{x / h} a_{l}(x) d x+h a_{f}(0) \leq h e^{t / h} a_{\max }-h a_{\max }+h a_{f}(0) \\
& \Leftrightarrow a_{\min }-e^{-t / h} a_{\min }+e^{-t / h} a_{f}(0) \leq \quad a_{f}(t) \quad \leq a_{\max }-e^{-t / h} a_{\max }+e^{-t / h} a_{f}(0) \\
& \Leftrightarrow a_{\min }+e^{-t / h}\left(a_{f}(0)-a_{\min }\right) \leq \quad a_{f}(t) \quad \leq a_{\max }+e^{-t / h}\left(a_{f}(0)-a_{\max }\right)
\end{aligned}
$$

Furthermore, if we assume that $a_{f}(0) \in\left[a_{\min }, a_{\max }\right]$, then:

$$
\left\{\begin{array}{l}
a_{f}(0)-a_{\max } \leq 0 \\
a_{f}(0)-a_{\min } \geq 0
\end{array}\right.
$$

Therefore, we have the following proposition:

Proposition 3 If $\forall t, r(t)=1, a_{l}(t) \in\left[a_{\min }, a_{\max }\right]$ and $a_{f}(0) \in\left[a_{\min }, a_{\max }\right]$, then $\forall t, a_{f}(t) \in$ $\left[a_{\min }, a_{\max }\right]$.

RR $n^{\circ} 3498$ 
Let us look closely at the condition on $a_{f}(0)$ :

$$
\begin{aligned}
& a_{\min } \leq a_{f}(0) \leq a_{\max } \\
\Leftrightarrow & a_{\min } \leq \frac{v_{l}(0)-v_{f}(0)}{h} \leq a_{\max } \\
\Leftrightarrow & h a_{\min } \leq v_{l}(0)-v_{f}(0) \leq h a_{\max }
\end{aligned}
$$

Equation (17) is actually an initial condition of the speed difference between the two vehicles, which is easy to check at the beginning of the simulation. In $[2,3]$ we made several simulations of an automated highway with multiple merge junctions. We choose $h=0.6 s$ to eliminate all accidents even in the high density case and to accommodate the merging traffic without causing too much congestion. With this value and with our chosen technology bounds, equation (17) becomes:

$$
-2.94 m s^{-1} \leq v_{l}(0)-v_{f}(0) \leq 1.18 m s^{-1}
$$

Now can we generalize proposition 3 to the cases $r(t)<1$ and $r(t)>1$ ? When $r(t) \neq 1$, the differential equation of the system is given by (6) instead of (15), so it is not integrable. Therefore, it is not possible to prove analytically that our result still holds when $r(t) \neq 1$ and we will have to rely on simulations: see Section 6 .

\section{$5 \quad$ Influence of the $\lambda$ parameter}

The condition $a_{f}(t)>-\lambda$ is a dynamic condition and we can make no assumption on $a_{f}(t)$ which depends on $v_{l}(t)$ and $p_{l}(t)$. We therefore rely on experimental results. As said in Section 4.3, we choose $h=0.6 \mathrm{~s}$. We use the SHIFT hybrid system simulator [5]. The specification of the leading vehicle is represented by the following hybrid automaton:

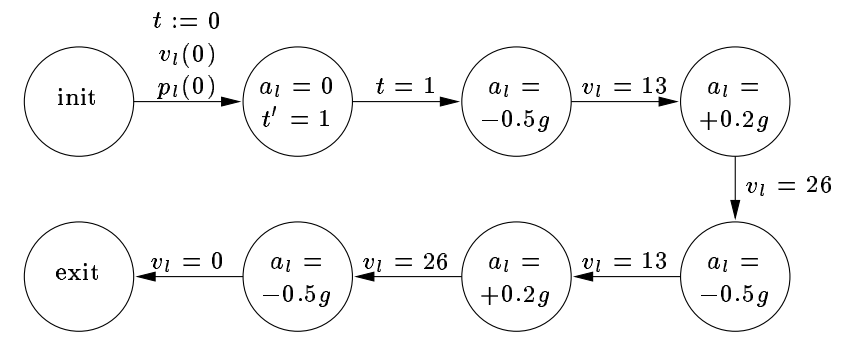

Figure 1: Hybrid automaton of the leading vehicle

Here we assume that the jerk of the leading vehicle is infinite. This behavior is among the worst possible cases. Indeed, the leading vehicle alternatively brakes and accelerates, making it hard, a priori, for the following vehicle to adjust its speed and position.

We first take the following initial conditions: 


$$
\left\{\begin{array} { l } 
{ p _ { l } ( 0 ) : = 1 0 m } \\
{ v _ { l } ( 0 ) : = 2 2 m s ^ { - 1 } }
\end{array} \quad \left\{\begin{array}{l}
p_{f}(0):=0 m \\
v_{f}(0):=26 m s^{-1}
\end{array}\right.\right.
$$

With these conditions, the initial value of the ration is $r(0)=\frac{10-0}{0.6 \times 26}=0.641$, which means that the following vehicle is too close. The corresponding evolution of the ratio, with values of $\lambda$ ranging from 3 to 17 is shown in Figure 2.

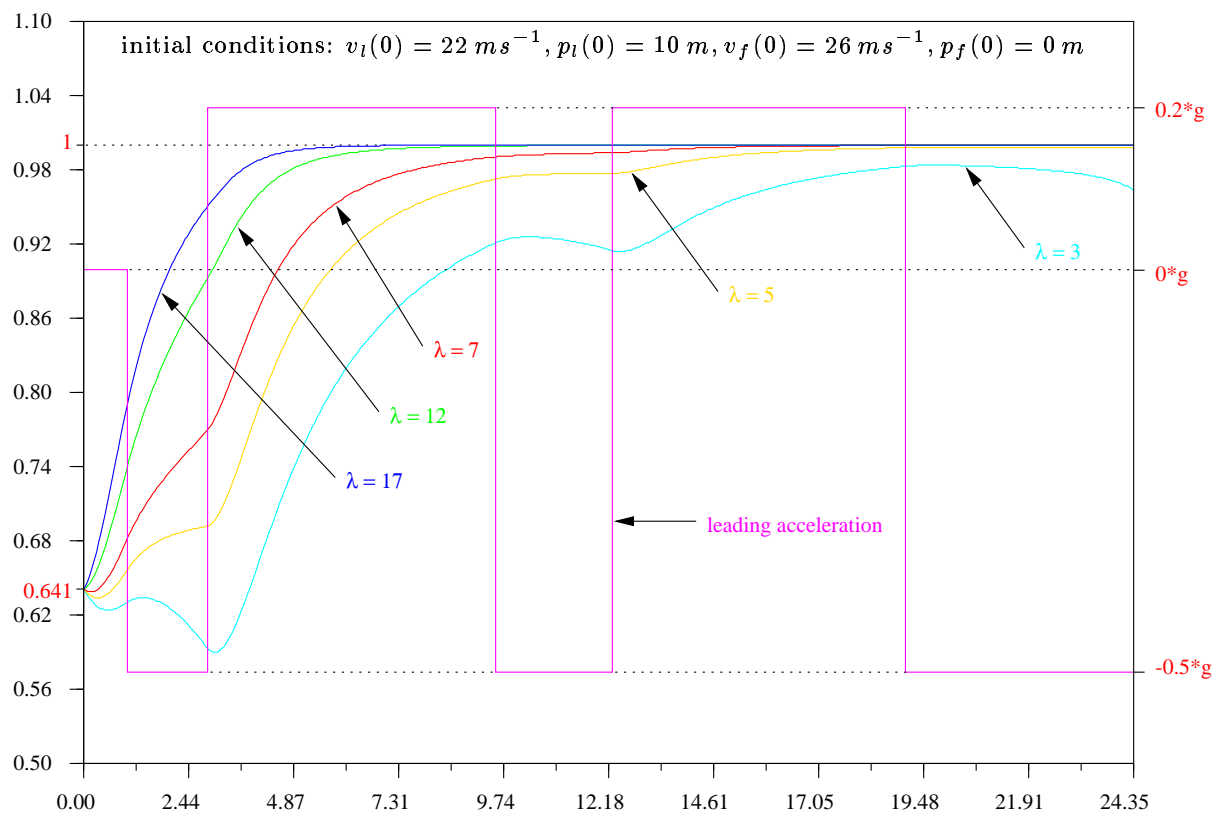

Figure 2: Evolution of the ratio function $t \mapsto r(t)$

As expected, the evolution of the ratio is influenced by the leading acceleration $a_{l}$. Concerning the influence of $\lambda$, just like we expected from our Houston highway simulations, the higher value is apparently the best. However, as we will see later, other factors have to be taken into account, like the saturation of the acceleration and the speed limit.

Now let us study when the derivative $r^{\prime}(t)$ changes its sign. Recall the condition which tells when $r^{\prime}(t)=0$ :

$$
r^{\prime}(t)=0 \Leftrightarrow a_{f}(t)+\lambda=0 \Leftrightarrow a_{f}(t)=-\lambda
$$

Note that $\lambda$ appears inside the definition of $a_{f}(t)$. Yet, condition (19) allows us to reason. A priori, $a_{f}(t)$ varies between $-\infty$ and $+\infty$. Each time $a_{f}(t)$ cuts the horizontal line $-\lambda$, there is a corresponding minimum or maximum of $r(t)$ along with a sign inversion of $r^{\prime}(t)$. Figure 3 shows the functions $a_{f}(t)$

$\mathrm{RR} \mathrm{n}^{\circ} 3498$ 
and $r(t)$. The function $r(t)$ is the same as in Figure 2. The horizontal line $-\lambda$ helps locating the maxima and minima of $r(t)$. Finally, the function $a_{l}(t)$ is drawn for comparison. As shown in Figure 2, for values of $\lambda$ greater than 7, there are no maxima and minima. This is easily understandable. Except at the beginning where $r(t) \ll 1$ and $v_{l}(t)-v_{f}(t) \ll 0, a_{f}(t)$ stays approximately between $-0.5 g$ and $+0.2 g$, which are the minimum and maximum of $a_{l}(t)$.

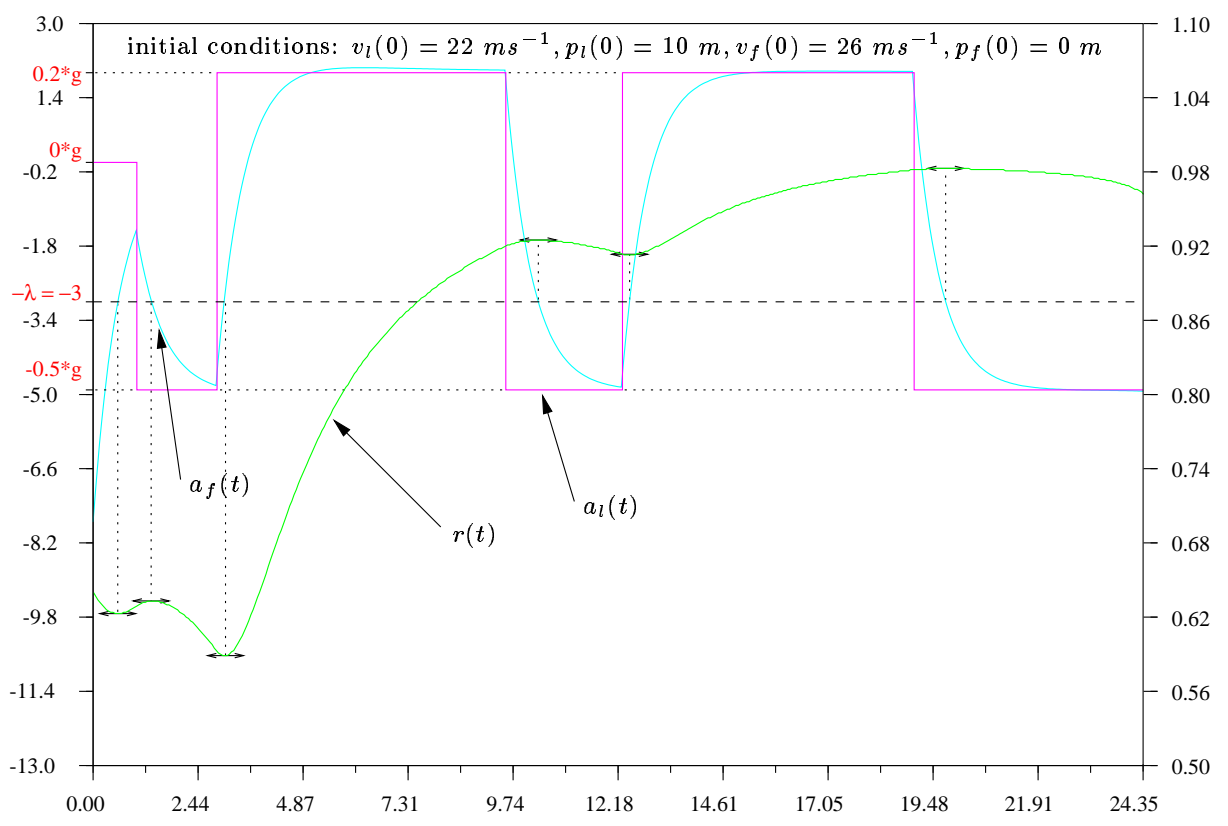

Figure 3: Maxima and minima of $r(t)$ for $\lambda=3$

We now take the following initial conditions:

$$
\left\{\begin{array} { l } 
{ p _ { l } ( 0 ) : = 2 0 m } \\
{ v _ { l } ( 0 ) : = 2 6 m s ^ { - 1 } }
\end{array} \quad \left\{\begin{array}{l}
p_{f}(0):=0 m \\
v_{f}(0):=22 m s^{-1}
\end{array}\right.\right.
$$

With these conditions, the initial value of the ration is $r(0)=\frac{20-0}{0.6 \times 22}=1.515$, which means that the following vehicle is too far away. The corresponding evolution of the ratio, again with values of $\lambda$ ranging from 3 to 17 is shown in Figure 4. 


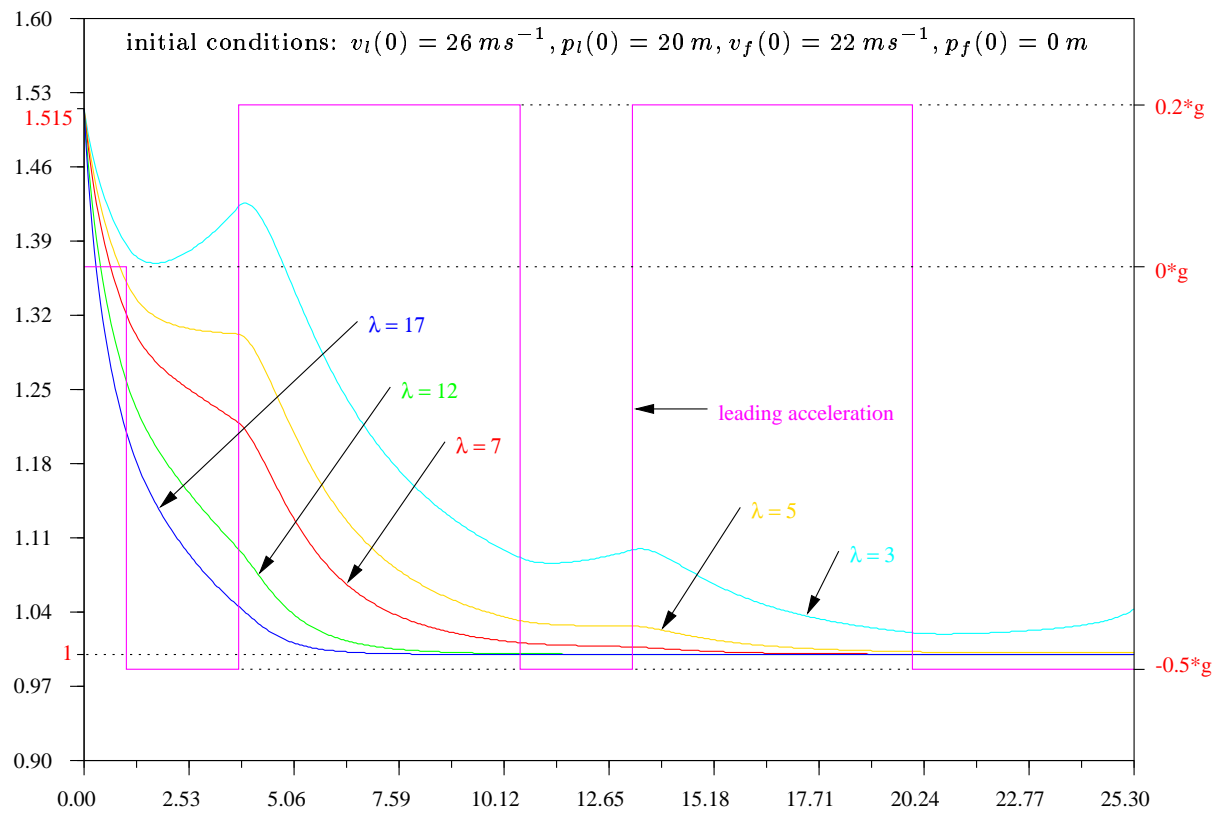

Figure 4: Evolution of the ratio function $t \mapsto r(t)$

The conclusions here are the same as with Figure 2. Of course this case is less crucial since collisions are less likely to occur when $r(t)>1$. We study in the next section the influence of the saturation, i.e., $a_{f}(t) \in[-0.5 g,+0.2 g]$, and we focus on the $r(t)<1$ case.

\section{Influence of the saturation $[-0.5 g,+0.2 g]$}

Figure 5 shows the acceleration of the following vehicle with the initial conditions of Figure 2 (only the cases $\lambda=3$ and $\lambda=17$ are traced). 


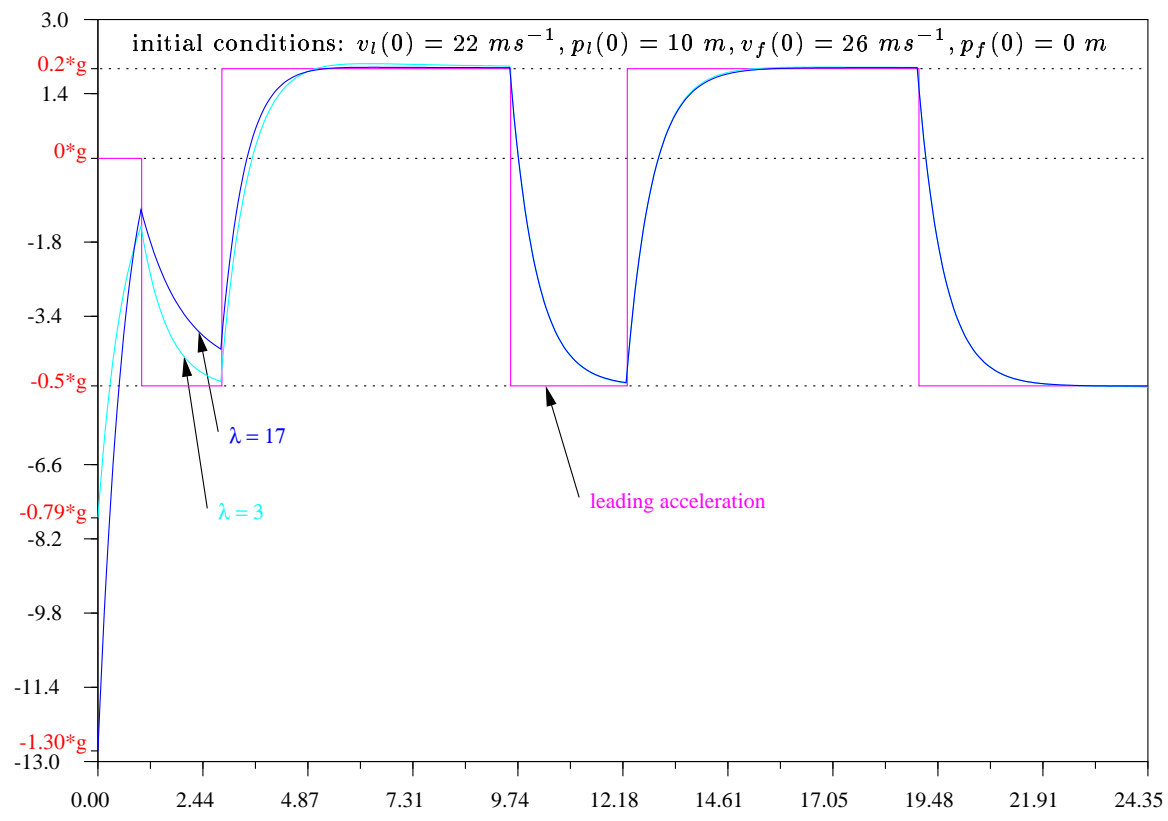

Figure 5: Acceleration of the following vehicle

For $\lambda=17$, the acceleration bounds are $[-12.77,+1.97]$ while for $\lambda=3$, they are $[-7.74,+2.05]$. This is well outside the technology bounds $[-0.5 g,+0.2 g]$ ! We now wish to take into account the technology bounds for the following vehicle, i.e., we saturate $a_{f}$. We thus modify the acceleration given by equation (4):

$$
\begin{aligned}
f(t)= & \lambda(r(t)-1)+\frac{v_{l}(t)-v_{f}(t)}{h} \\
a_{f}(t)= & \text { if } f(t)>0.2 g \text { then } 0.2 g \\
& \text { else if } f(t)<-0.5 g \text { then }-0.5 g \\
& \text { else } f(t)
\end{aligned}
$$

We perform the same simulations as in Figure 2. The result is shown in Figure 6 . 


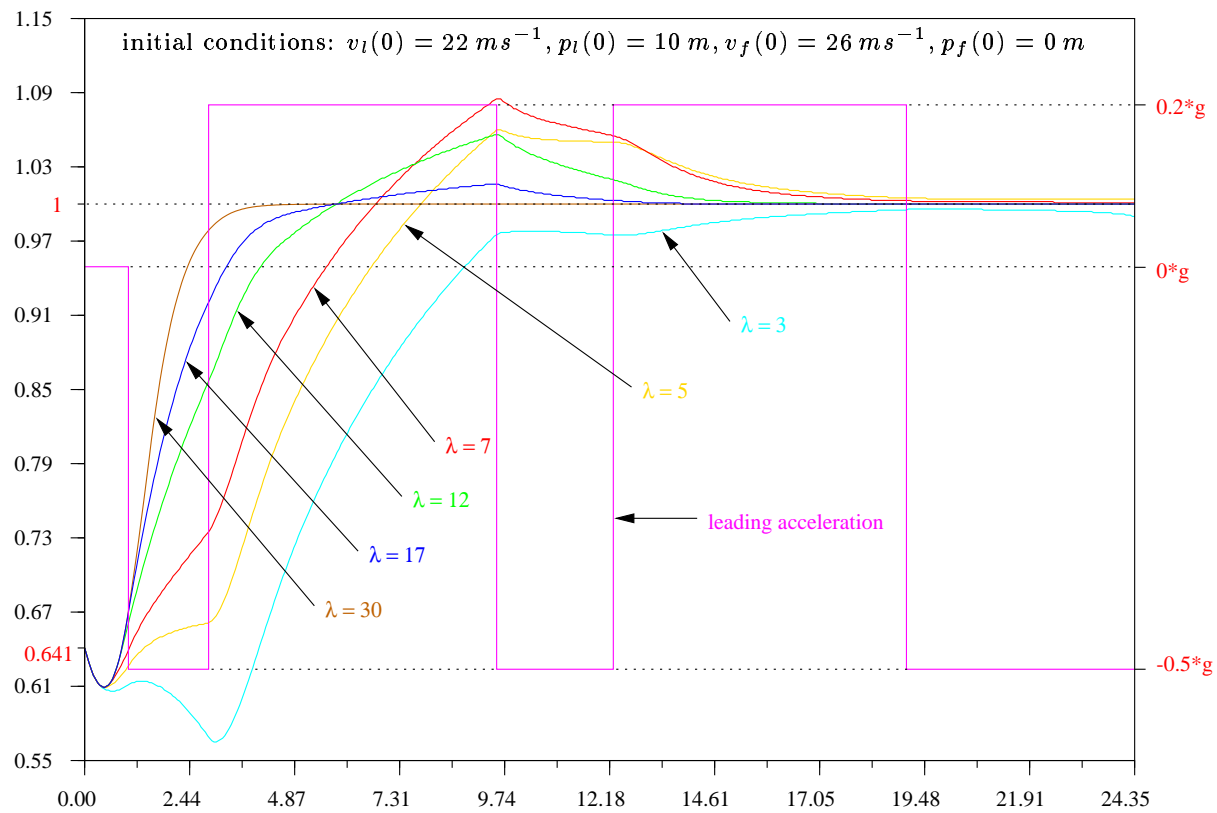

Figure 6: Evolution of the ratio in the saturated case

Clearly, the result given in proposition 1 does not hold anymore: for $\lambda=17$, the function $r(t)$ crosses the horizontal line $r=1$ ! Indeed, when the leading vehicle accelerates, the following vehicle can't catch up because his acceleration is limited to $a_{\max }$. Therefore the gap increases to the point where the ratio becomes greater than 1 . Yet, the ratio does not oscillate around the value 1 , and even in the saturated case the system is asymptotically stable. Finally, note that the greatest value of $\lambda$ still provides the best behavior for the following vehicle. For $\lambda=30$, the ratio reaches even faster its limit 1 and does not even become greater than 1 .

Moreover, as stated in Section 4.3, when $r(t)$ is close enough to 1, proposition 3 is valid. For $\lambda=17$, $\forall t>13.4 s$, we have $|1-r(t)|<0.01$ and indeed, $a_{f}(t) \in[-0.5 g,+0.2 g]$. Of course the value after which $r(t)$ is considered to be close enough to 1 depends on $\lambda$.

\section{Decoupling and linearization}

We propose in this final Section a new acceleration law. The technique used here is inspired from the decoupling and feedback linearization well known in the automatic control field.

The idea is to choose $a_{f}(t)$ such that the ratio $r(t)$ converges exponentially towards 1 . Hence we want:

$$
r(t)=1+r(0) e^{-\lambda t}
$$


This yields:

$$
r^{\prime}(t)=-\lambda r(0) e^{-\lambda t}=-\lambda(r(t)-1)
$$

Now from equation (1) we compute:

$$
\begin{aligned}
r^{\prime}(t) & =\frac{h v_{f}(t)\left(v_{l}(t)-v_{f}(t)\right)-h a_{f}(t)\left(p_{l}(t)-p_{f}(t)\right)}{h^{2} v_{f}(t)^{2}} \\
& =\frac{v_{l}(t)-v_{f}(t)}{h v_{f}(t)}-\frac{a_{f}(t) r(t)}{v_{f}(t)}
\end{aligned}
$$

By combining equations (22) and (23), we get:

$$
\begin{aligned}
& \frac{v_{l}(t)-v_{f}(t)}{h v_{f}(t)}-\frac{a_{f}(t) r(t)}{v_{f}(t)}=-\lambda(r(t)-1) \\
\Leftrightarrow & \frac{a_{f}(t) r(t)}{v_{f}(t)}=\frac{v_{l}(t)-v_{f}(t)}{h v_{f}(t)}+\lambda(r(t)-1) \\
\Leftrightarrow & a_{f}(t)=\frac{v_{f}(t)}{r(t)}\left(\frac{v_{l}(t)-v_{f}(t)}{h v_{f}(t)}+\lambda(r(t)-1)\right) \\
\Leftrightarrow & a_{f}(t)=\frac{v_{l}(t)-v_{f}(t)}{h r(t)}+\lambda v_{f}(t)\left(1-\frac{1}{r(t)}\right)
\end{aligned}
$$

Now with the acceleration law of equation (24), propositions 1 and 2 are trivial to prove thanks to the exponential convergence. Moreover, proposition 3 still holds since $r(t)=1$ yields

$$
a_{f}(t)=\frac{v_{l}(t)-v_{f}(t)}{h}+\lambda v_{f}(t)(1-1)=\frac{v_{l}(t)-v_{f}(t)}{h}
$$

which is the same as equation (12).

For the same value of $\lambda$, the "new" acceleration law, given by equation (24), is much better than the "old" one, of equation (4), even in the truncated case. For instance, for $\lambda=17$ with non truncated accelerations, we get: 


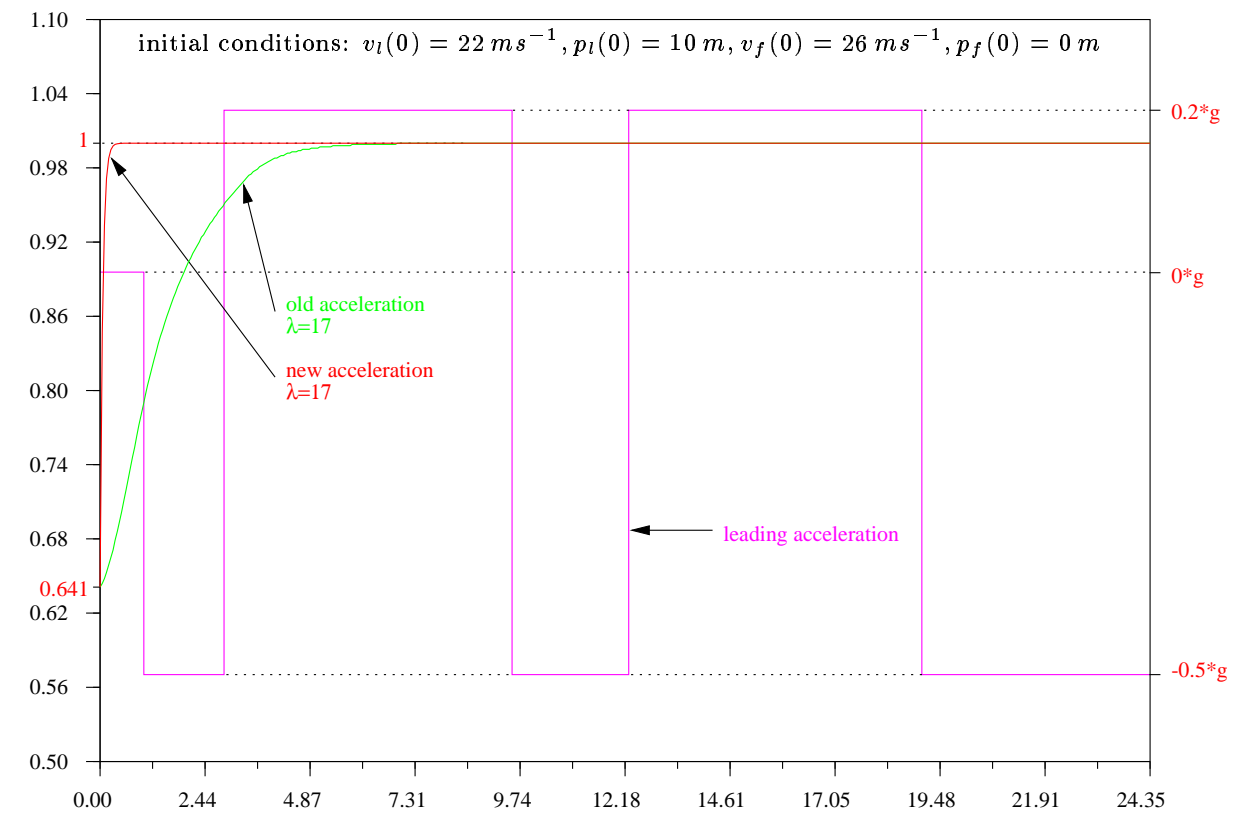

Figure 7: Old law versus new law (non truncated)

In the truncated case, we have added the the curve of the old acceleration obtained for $\lambda=30$ : 


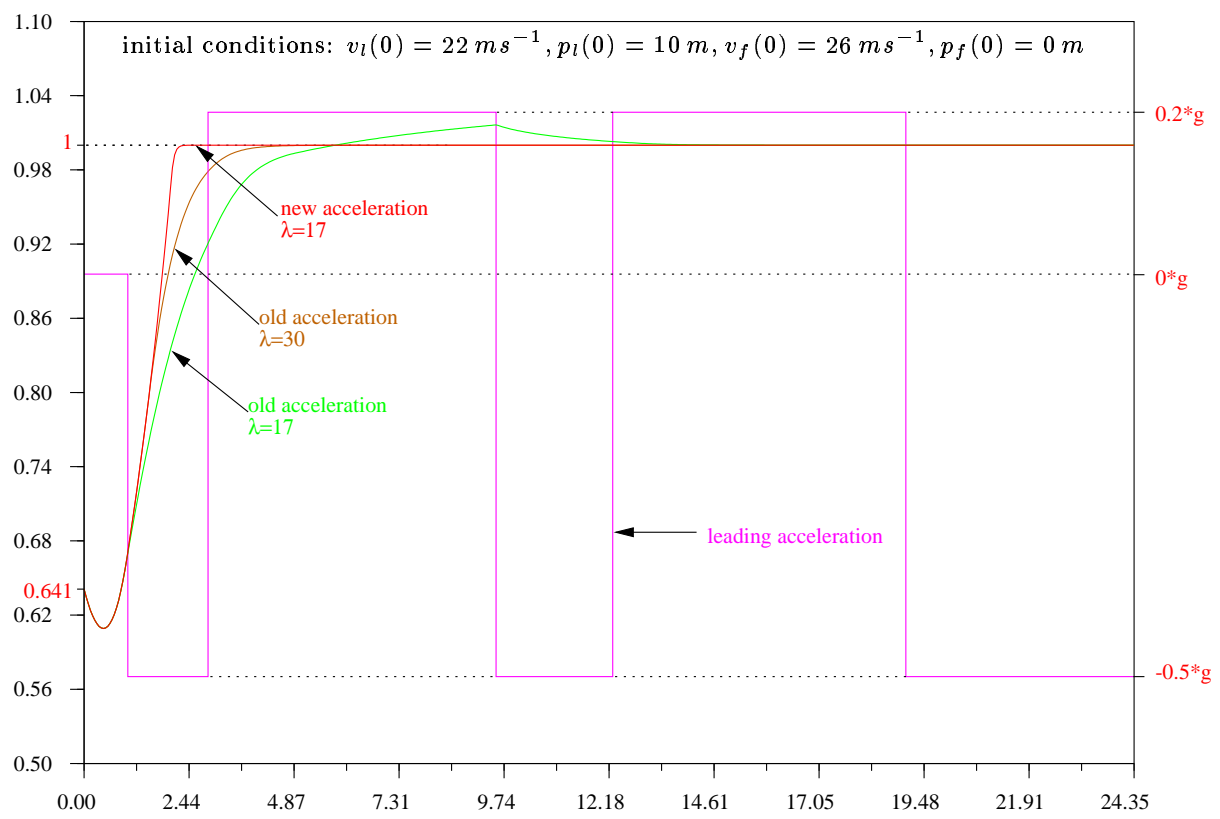

Figure 8: Old law versus new law (truncated)

We could conclude at this point that the new acceleration law is much better than the new acceleration law. However this comparison is merely a question of gain. For instance, the old acceleration with $\lambda=30$ is very close to the new acceleration with $\lambda=17$. Moreover, if we take into account the maximum jerk, then both laws are comparable.

\section{Conclusion}

We have studied in this article the convergence of an acceleration law for an autonomous vehicle. The following vehicle must maintain a constant time headway behind a leading vehicle which alternates hard breaking and hard acceleration. We compute a ratio which measures how accurately is the following vehicle positioned with respect to the leading vehicle. The acceleration law is designed by taking into account the positioning error as well as the relative speed error. Both informations can be made available to the following vehicle only through sensors, i.e., no inter-vehicle communication is needed.

We have first studied this acceleration law analytically in the ideal case where the acceleration is not limited to any technology bounds. We have proved two major results. The first one states that under some dynamic condition on the leading acceleration, the ratio converges towards 1 , which means that the following vehicle never collides with the leading vehicle. The second result states that when the ratio is equal to 1 , if the leading acceleration is bounded, then the following acceleration is also bounded and by the same bounds. 
Then we have performed extensive simulations to understand the influence of the gain parametering the acceleration law as well as the technology bounds on the acceleration. These simulations show to which extent our results are valid in the non ideal case, i.e., when the acceleration is saturated.

Finally, we have applied decoupling and feedback linearization techniques to design a new acceleration law for which the ratio is guaranteed to converge exponentially towards 1 . The simulations performed with this new acceleration law show that in the non ideal case, both laws are comparable provided that we take a greater gain for the old law than for the new one.

\section{Acknowledgment}

Many thanks to John Lygeros for showing us how to obtain the new acceleration law with decoupling and feedback linearization techniques.

\section{References}

[1] Nahsc milestone 2 report (task c2: Downselect system configuration and workshop \#3). Internal NAHSC document to be submitted to the U.S. Department of Transportation in 1997 as a formal program deliverable, 1996.

[2] M. Antoniotti, A. Deshpande, and A. Girault. Microsimulation analysis of a hybrid system model of multiple merge junction highways and semi-automated vehicles. In IEEE International Conference on Systems Man and Cybernetics, SMC'97, Orlando, FL, October 1997. IEEE press.

[3] M. Antoniotti, A. Deshpande, and A. Girault. Microsimulation analysis of multiple merge junctions under autonomous ahs operation. In IEEE Conference on Intelligent Transportation Systems, ITSC'97, Boston, MA, November 1997. IEEE press.

[4] P. Daviet and M. Parent. Platooning techniques for empty vehicle distribution in the PraxitèLE project. In Proceeding of the 4 th Mediterranean Symposium on New Directions in Control and Automation, Maleme, Grece, June 1996.

[5] A. Deshpande, A. Göllü, and L. Semenzato. The ShIFT programming language for dynamic networks of hybrid automata. IEEE Transactions on Automatic Control, 43(4):584-588, April 1998. Research Report UCB-ITS-PRR-97-7.

[6] S. E. Shladover. Review of the state of development of advanced vehicle control systems (avcs). Vehicle System Dynamics, 24:551-595, 1995.

[7] K.J. Åström and S. Brufani. Manual control of an unstable system with a saturating actuator. In $I E E E$ Conference on Decision and Control, CDC'97, pages 964-965, San Diego, CA, December 1997. IEEE Press.

[8] P. Varaiya. Smart cars on smart roads: Problems of control. IEEE Transactions on Automatic Control, 38(2):195-207, February 1993.

[9] H. Ye, A.N. Michel, and L. Hou. Stability theory for hybrid dynamical systems. IEEE Transactions on Automatic Control, 43(4):461-474, April 1998.

$\mathrm{RR} \mathrm{n}^{\circ} 3498$ 


\section{Annex: Proof of proposition 1}

Our induction hypothesis is that the $n^{\text {th }}$ derivative is:

$$
\begin{aligned}
r^{(n)}(t) & =\frac{(1-r(t)) X_{n-1}^{0}(t)+\sum_{i=1}^{n-1} r^{(i)}(t) X_{n-1}^{i}(t)}{v_{f}(t)^{2^{n-1}}} \\
& =\frac{(1-r(t)) X_{n-1}^{0}(t)+r^{\prime}(t) X_{n-1}^{1}(t)+\cdots+r^{(n-1)}(t) X_{n-1}^{n-1}(t)}{v_{f}(t)^{2^{n-1}}}
\end{aligned}
$$

Let us compute the $n+1^{\text {th }}$ derivative:

$$
\begin{aligned}
r^{(n+1)}(t)= & \frac{1}{v_{f}(t)^{2^{n}}}\left[-r^{\prime}(t) X_{n-1}^{0}(t) v_{f}(t)^{2^{n-1}}+\right. \\
& (1-r(t)) X_{n-1}^{0}{ }^{\prime}(t) v_{f}(t)^{2^{n-1}}+ \\
& r^{\prime \prime}(t) X_{n-1}^{1}(t) v_{f}(t)^{2^{n-1}}+ \\
& r^{\prime}(t) X_{n-1}^{1}{ }^{\prime}(t) v_{f}(t)^{2^{n-1}}+\cdots+ \\
& r^{(n)}(t) X_{n-1}^{n-1}(t) v_{f}(t)^{2^{n-1}}+ \\
& r^{(n-1)}(t) X_{n-1}^{n-1}(t) v_{f}(t)^{2^{n-1}}- \\
& 2^{n-1} v_{f}(t)^{2^{n-1}-1} a_{f}(t)(1-r(t)) X_{n-1}^{0}(t)- \\
& 2^{n-1} v_{f}(t)^{2^{n-1}-1} a_{f}(t) r^{\prime}(t) X_{n-1}^{1}(t)-\cdots- \\
& \left.2^{n-1} v_{f}(t)^{2^{n-1}-1} a_{f}(t) r^{(n-1)}(t) X_{n-1}^{n-1}(t)\right] \\
= & \frac{(1-r(t)) X_{n}^{0}(t)+\sum_{i=1}^{n} r^{(i)}(t) X_{n}^{i}(t)}{v_{f}(t)^{2^{n}}}
\end{aligned}
$$

where

$$
\left\{\begin{array}{lll}
X_{n}^{0}(t)= & X_{n-1}^{0}{ }^{\prime}(t) v_{f}(t)^{2^{n-1}}-2^{n-1} v_{f}(t)^{2^{n-1}-1} a_{f}(t) X_{n-1}^{0}(t) \\
X_{n}^{1}(t)= & X_{n-1}^{1}(t) v_{f}(t)^{2^{n-1}}-X_{n-1}^{0}(t) v_{f}(t)^{2^{n-1}}- \\
& \\
& 2^{n-1} v_{f}(t)^{2^{n-1}-1} a_{f}(t) X_{n-1}^{1}(t) \\
\vdots & \\
X_{n}^{n-1}(t)= & X_{n-1}^{n-1}(t) v_{f}(t)^{2^{n-1}}+\cdots+2^{n-1} v_{f}(t)^{2^{n-1}-1} a_{f}(t) X_{n-1}^{n-1}(t) \\
X_{n}^{n}(t)= & X_{n-1}^{n-1}(t) v_{f}(t)^{2^{n-1}}
\end{array}\right.
$$




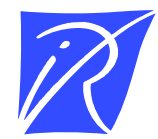

Unit 'e de recherche INRIA Lorraine, Technopôle de Nancy-Brabois, Campus scientifique, 615 rue du Jardin Botanique, BP 101, 54600 VILLERS LÈS NANCY

Unit'e de recherche INRIA Rennes, Irisa, Campus universitaire de Beaulieu, 35042 RENNES Cedex Unit'e de recherche INRIA Rhône-Alpes, 655, avenue de l'Europe, 38330 MONTBONNOT ST MARTIN Unit'e de recherche INRIA Rocquencourt, Domaine de Voluceau, Rocquencourt, BP 105, 78153 LE CHESNAY Cedex Unit'e de recherche INRIA Sophia-Antipolis, 2004 route des Lucioles, BP 93, 06902 SOPHIA-ANTIPOLIS Cedex

\section{Éditeur}

INRIA, Domaine de Voluceau, Rocquencourt, BP 105, 78153 LE CHESNAY Cedex (France) http://www.inria.fr

ISSN 0249-6399 